\title{
Aplicações do laser de baixa intensidade no tratamento da síndrome do túnel do carpo
}

\author{
João Paulo Caldas Cardozo' \\ Léo Sebastião David Filho \\ Hugo Alves de Souza ${ }^{3}$ \\ Flavia Ladeira Ventura Dumas ${ }^{4}$
}

\section{Resumo}

O objetivo deste trabalho foi analisar, por meio de revisão bibliográfica, a eficiência da aplicação do Laser de baixa intensidade (LBI) no tratamento da Síndrome do Túnel do Carpo (STC). Para isso foi realizada uma pesquisa de artigos nas bases de dados PUBMED e BIREME, publicados entre 1985 e 2009, nos idiomas espanhol, inglês e português. Grande parte dos artigos consultados revelou que a LBI é eficaz no tratamento de pacientes diagnosticados com Síndrome do Túnel do Carpo. Concluiu-se que, quando associada ao uso de órtese para o punho, a laserterapia é um método eficiente no tratamento da síndrome do túnel do carpo.

Palavras-chave: Síndrome do Túnel do Carpo. Laserterapia. Compressão do nervo mediano.

\footnotetext{
${ }^{1}$ Extensionista do Plano de Incentivo à Extensão/UniCEUB - Fisioterapia Ambulatorial na ADESU. Graduando do curso de Fisioterapia do UniCEUB. E-mail: joaofisiocaldas@ gmail.com

2 Extensionista do Plano de Incentivo à Extensão/UniCEUB - Fisioterapia Ambulatorial na ADESU. Graduando do curso de Fisioterapia do UniCEUB. E-mail: leodavidfilho@gmail. com

${ }^{3}$ Graduação em Fisioterapia pelo Centro Universitário de Brasília (2006) e mestrado em Ciências da Saúde pela Universidade de Brasília (2009). Docente do curso de Fisioterapia do Centro Universitário de Brasília. E-mail: hugoanatomia@gmail.com

${ }^{4}$ Graduação em Fisioterapia pela Universidade Católica de Brasília (2002) e mestrado em Engenharia Biomédica pela Universidade do Vale do Paraíba (2004). Docente do curso de Fisioterapia do Centro Universitário de Brasília e fisioterapeuta da Secretaria de Saúde do Distrito Federal. E-mail: fladeira@gmail.com
} 


\section{Introdução}

A Síndrome do Túnel do Carpo (STC) é a neuropatia compressiva com maior índice de acometimento nos seres humano (KAROLCZAC; VAZ; MERLO, 2005). Trata-se de uma patologia multifatorial provocada pela compressão do nervo mediano no seu trajeto através do túnel carpiano. Os sinais e sintomas mais reclamados entre os que sofrem dessa compressão são: dor, alterações sensitivas e motoras, parestesia, edema, inflamação e atrofia tênar (KOUYOUMDJIAN, 1999; KAROLCZAC; VAZ; MERLO, 2005; BRAZ et al., 2008; DINCER et al., 2009).

Segundo Karolczak, Vaz e Merlo (2005), o diagnóstico preciso da STC é difícil e consiste basicamente em exame físico e eletroneuromiográfico. Alguns autores sugerem que, associado à eletroneuromiografia (ENMG), sejam realizados os Testes Especiais de Phalen e Tinel Hofmann para confirmação do diagnóstico de STC (KAROLCZAC; VAZ; MERLO, 2005). Karolczak, Vaz e Merlo (2005) afirmam que, por si só, os testes produzem um alto número de resultados falso-positivos e falso-negativos, por isso a sugestão da ENMG conjunta.

De acordo com Veçoso (1993), 75\% dos casos de STC se dão em mulheres. Outros estudos citam informações semelhantes, como os de Kouyoumdjian (1999), Evcik et al. (2007) e Braz et al. (2008), e acrescentam que a patologia é mais incidente em mulheres acima dos 30 anos. A STC é uma frequente complicação durante o período gestacional, acometendo cerca de $62 \%$ da população, segundo o estudo realizado por Ablove e Ablove (2009), se mostrando uma síndrome nervosa muito comum durante esta fase da vida, e que está relacionada, principalmente, ao aumento do líquido extracelular. Apesar de não haver consenso, a obesidade, atividades motoras repetitivas e algumas patologias sistêmicas contribuem para o aparecimento da STC (KAROLCZAC; VAZ; MERLO, 2005).

São atribuídas ao laser de baixa intensidade algumas propriedades terapêuticas, como efeitos analgésicos, anti-inflamatórios e fibrinolíticos (BRAZ et al., 2008). Segundo Kouyoumdjian (1999), Karolczak; Vaz; Merlo (2005), Evcik et al. (2007), Braz et al. (2008) e Dincer et al. (2009), a laserterapia é uma medida conservadora eficaz no tratamento da STC. Na fisioterapia, os lasers mais utilizados são o Hélio-Neônio (HeNe) e o Arsianeto de Gálio (AsGa), sendo o primeiro, com potencial terapêutico elevado, para lesões superficiais, como as dermatológicas, e 
o segundo, mais indicado para lesões mais profundas, dos tipos articular e óssea (VEÇOSO, 1993; STARKEY, 2001).

Sabe-se hoje que a ação do laser não é igualmente positiva em todos os pacientes (VEÇOSO, 1993). Uma das explicações para isso é o fator individualidade de cada um, este que é determinante nos resultados de sua utilização. Pessoas possuem quantidades diferentes de células absorventes, além da disposição espacial delas. Até mesmo se considerarmos uma única pessoa, as suas diferentes regiões corporais apresentam índices de absorção variados. Outro fator pouco citado em artigos científicos é a tonalidade da pele dos pacientes. Indivíduos de peles mais escuras absorvem maior quantidade de radiação em função da maior quantidade de pigmentos; logo, é de grande importância a presença de tal informação num estudo, visto que se trata de um fator de grande influência sobre os resultados finais (VEÇOSO, 1993).

A utilização do laser de baixa intensidade como forma de tratamento da STC ainda deixa algumas dúvidas com relação à sua eficiência, mas o número de estudos na área é crescente. Este estudo de revisão se mostra relevante devido à prevalência e às incidências cada vez maiores de casos de STC no país e no mundo, principalmente nas classes trabalhadoras inferiores (KAROLCZAC; VAZ; MERLO, 2005; DINCER et al., 2009).

Este trabalho de revisão tem por objetivo analisar, por meio da literatura e de artigos científicos, a eficiência do laser de baixa intensidade no tratamento da STC.

\section{Metodologia}

Foram consultadas as bases de dados PUBMED e BIREME, utilizando os termos "carpal tunnel syndrome", "laser" e "median nerve compression" e seus correspondentes em português e espanhol. Foram selecionados 21 artigos com base na leitura dos respectivos resumos. Após leitura na íntegra, foram incluídos no estudo os artigos que apresentavam ensaios clínicos randomizados e estudos longitudinais prospectivos, e excluídos aqueles que apresentavam apenas o laser cirúrgico como forma de tratamento da STC, bem como os estudos de caso, reduzindo o número 
para 12 artigos, os quais foram utilizados. Os artigos utilizados são datados de 1985 a 2009, nos idiomas espanhol, inglês e português.

\section{Resultado/discussão}

A aplicação do laser de baixa intensidade no tratamento da Síndrome do Túnel do Carpo não é uma técnica recente. Muitos são os estudos publicados que experimentaram e provaram a eficácia desse tratamento; porém, ainda não se chegou a um consenso sobre os parâmetros de utilização do laser para que os melhores resultados possam ser obtidos. Mesmo com essas divergências, o laser tem se mostrado positivo no tratamento da STC, especialmente em casos agudos, como demonstrou o estudo de Elwakil et al. (2007). Tal dado é de grande importância, visto que, até pouco tempo atrás, a cirurgia era o único método disponível para realizar a descompressão do nervo mediano ao longo do túnel carpiano (KAROLCZAC; VAZ; MERLO, 2005).

Braz et al. (2008), num estudo longitudinal prospectivo, contaram com 10 pacientes, todos diagnosticados com STC, de ambos os gêneros, 9 do feminino e 1 do masculino, com idade média de 30 anos. Neste estudo foi utilizada uma forma indireta de aplicação do laser, onde este não foi aplicado diretamente no local acometido, neste caso no túnel carpiano, e sim na região cervical da coluna vertebral. Ao irradiar o laser de forma contínua, sobre a região cervical, entre as vértebras C2 e C7, percebeu-se resultados positivos significativos no local lesionado, mostrando que a irradiação do laser na origem do nervo também é positiva. Uma das explicações dada pelos autores para tal fato é que o cromóforo de maior absorção é a mitocôndria, e esta é mais numerosa no corpo celular e dendritos do que nos axônios, portanto, quando irradiado na região vertebral, o laser parece ser mais bem absorvido. O tratamento foi realizado durante 5 dias consecutivos e cada sessão teve duração média de 30 minutos. O método de utilização do laser de Arsianeto de Gálio e Alumínio (AsGaAl) foi de irradiação contínua sobre a região cervical, de C2 a C7, utilizando técnica pontual com 12 pontos de aplicação no trajeto das vértebras citadas. A densidade de energia utilizada foi de $80 \mathrm{~J} / \mathrm{cm}^{2}$, a potência de $120 \mathrm{~mW}$ e o comprimento de onda de $830 \mathrm{~nm}$. Apesar de não apresentar dados estatísticos referentes ao número de pacientes que relataram melhora pós-interven- 
ção, foi observado que em todos os pacientes houve diminuição da dor e melhora do padrão funcional e das atividades na vida diária, fatos comprovados por meio de questionário de avaliação da dor, mostrando a eficiência do tratamento nestes pacientes. Braz et al. (2008) obtiveram ainda um importante achado neste estudo. A densidade de energia do laser utilizado também chama atenção. Enquanto os demais autores utilizaram o laser com densidade de energia entre $1 \mathrm{~J} / \mathrm{cm}^{2} \mathrm{e} 7 \mathrm{~J} / \mathrm{cm}^{2}$, Braz et al. (2008), utilizaram o laser com $80 \mathrm{~J} / \mathrm{cm}^{2}$, valor extremo observado em toda literatura revisada, porém, Kitchen (2003) comenta que os valores típicos para os tratamentos de rotina podem variar de menos de 1 para mais de $30 \mathrm{~J} / \mathrm{cm}^{2}$. Outro motivo que justifica a utilização de tamanha densidade de energia está embasado no fato da aplicação indireta do laser exigir uma mais densidade de energia maior para que esta demonstre resultados efetivos no local acometido (KITCHEN, 2003).

O trabalho de Elwakil et al. (2007), um estudo longitudinal prospectivo, teve por objetivo comparar os resultados do tratamento da STC por laserterapia em relação aos obtidos pelo tratamento cirúrgico. Participaram do estudo 54 pacientes divididos em 2 grupos iguais. O Grupo 1 foi submetido a laserterapia de $\mathrm{HeNe}$, com densidade de energia de $3 \mathrm{~J} / \mathrm{cm}^{2}$, densidade de potência mínima de $12 \mathrm{~mW}$ e o comprimento de onda de $632,8 \mathrm{~nm}$. O tempo de duração foi estabelecido pelo aparelho utilizado. Os pacientes foram tratados duas vezes por semana, durante seis semanas, com um total de 12 sessões. O grupo 2 foi submetido a tratamento cirúrgico. A laserterapia, apesar de exigir mais tempo e dedicação do paciente, se mostrou mais eficaz que a cirurgia, principalmente no que diz respeito à diminuição da dor, que para muitos é o maior desconforto provocado pela compressão do nervo mediano. A cirurgia se mostrou positiva na diminuição da parestesia, mas por outro lado, a dor persistiu por algum tempo, por conta do estresse cirúrgico, não sendo totalmente eficiente. Sendo assim, o laser de baixa intensidade demonstrou ser um tratamento eficiente para a STC, especialmente em casos agudos.

Numa outra investigação Ekim et al. (2007) avaliaram os efeitos da terapia de laser de baixa intensidade sobre a dor, capacidade funcional e força da preensão palmar de pacientes com artrite reumatóide e STC. Para isso, utilizaram o Questionário de Boston de sintomas severos, o Questionário de Boston de status funcional e o teste de dinamometria da preensão palmar. Foram selecionados para o estudo 19 pacientes diagnosticados com STC. Esses pacientes foram divididos aleatoria- 
mente em 2 grupos de tratamento. O grupo 1 foi submetido a laserterapia de As$\mathrm{GaAl}$, com densidade de energia de $1,5 \mathrm{~J} / \mathrm{cm}^{2}$, densidade de potência de $50 \mathrm{~mW}$ e comprimento de onda de $780 \mathrm{~nm}$, enquanto o grupo 2 foi submetido a sessões de laser placebo. Ambos os grupos foram tratados durante 10 dias, sendo 1 sessão diária. Tanto a laserterapia de baixa intensidade quanto o laser placebo demonstraram resultados positivos, sendo o laser de baixa intensidade mais eficiente para o tratamento da Dor e na melhora da Capacidade funcional, segundo Questionário de Boston de Status Funcional e Escala Visual Analógica de Dor.

Em estudo realizado por Evcik et al. (2007), foram comparados os resultados da aplicação da laserterapia em relação ao tratamento placebo da mesma técnica em pacientes diagnosticados com STC. Para confirmação da presença de STC nos pacientes foram realizados: testes clínicos, sendo estes os testes de Tinel Hofmann, Phalen, Buda e Flick; teste de força bilateral, realizados com dinamômetros; testes de dor, a partir de Escava Visual Analógica (EVA) realizados dia e noite; testes de capacidade funcional; e Eletrodiagnóstico. Participaram do estudo 81 pacientes de ambos os gêneros, sendo 70 do feminino e 11 do masculino, com idades entre 26 e 78 anos. Este foi um estudo randomizado duplo cego com grupo controle placebo, onde os pacientes foram divididos em 2 grupos: o grupo 1, composto por 41 pacientes, foi submetido a laserterapia de baixa intensidade, enquanto o grupo 2, composto por 40 pacientes, foi submetido a aplicações placebo de laserterapia, representando o grupo controle do estudo. No grupo 1 foi utilizado o laser de AsGaAl, cujo comprimento de onda é $830 \mathrm{~nm}$, técnica de irradiação pontual perpendicular a pele em 2 pontos da área do túnel cárpico, densidade de energia de $7 \mathrm{~J} / \mathrm{cm}^{2}$, densidade de potência de $450 \mathrm{~mW}$, com tempo de aplicação de 2 minutos por ponto. No grupo 2 foi realizado o placebo da laserterapia com técnica e tempo de aplicação iguais aos do grupo 1. Durante o estudo, todos os pacientes utilizaram uma órtese no punho acometido com STC enquanto dormiam. Em ambos os grupos realizaram-se 10 sessões, sendo 1 sessão diária. Em relação à dor e a capacidade funcional dos pacientes não foram observadas melhoras significativas nos dois grupos. No que diz respeito à força bilateral, o grupo submetido à laserterapia apresentou melhores resultados.

Equipamentos mais modernos de laserterapia permitem ao terapeuta modular o laser de várias formas, o que dificulta a formação de um consenso sobre 
os parâmetros de utilização deste para os variados casos a que se aplica, além de provocar algumas divergências entre autores. Essa divergência pode ser observada ao comparar dois estudos: Ekim et al. (2007), aplicaram o laser AsGaAl, com densidade de energia de $1,5 \mathrm{~J} / \mathrm{cm}^{2}$, densidade de potência de $50 \mathrm{~mW}$ e comprimento de onda de 780nm, enquanto Evcik et al. (2007), aplicaram o laser AsGaAl, com densidade de energia de $7 \mathrm{~J} / \mathrm{cm}^{2}$, densidade de potência de $450 \mathrm{~mW}$ e comprimento de onda de $830 \mathrm{~nm}$. Apesar da modulação totalmente diferente nos estudos citados, foram observados resultados positivos em ambos. Outro ponto a ser observado no exemplo é que o laser utilizado pelos dois foi o mesmo, AsGaAl, e que apesar da coincidência houve uma divergência no comprimento de onda informado por cada autor.

Em estudo desenvolvido por Bakhtiary e Rashidy-Pour (2004), comparouse a eficácia do ultrasom e da Laserterapia de baixa intensidade no tratamento da STC moderada ou grave. Para realização do mesmo, os autores contaram com 50 pacientes diagnosticados a partir de eletromiografia com STC uni ou bilateral, somando 90 mãos acometidas. Dividiu-se a amostra em dois grupos de forma que ficassem 45 mãos em cada grupo. O grupo 1 recebeu aplicações do ultrasom terapêutico com transdutor de $5 \mathrm{~cm}^{2}$, frequencia de $1 \mathrm{MHz}$ e intensidade de 1.0 $\mathrm{W} / \mathrm{cm}^{2}$ no modo pulsado com proporção 1:4 durante 3 semanas, sendo 5 sessões semanais. Já o grupo 2 recebeu laserterapia de baixa intensidade, onde aplicou-se Laser-Diodo com comprimento de onda de 830nm e densidade de energia de 9J/ $\mathrm{cm}^{2}$ em 5 pontos pelo túnel carpiano durante 3 semanas, sendo 5 sessões semanais.

O diferencial do estudo supracitado se fundamenta na iniciativa dos autores de quantificarem não só a redução da dor, mas também em qual grupo houve maior ganho de força a partir dos testes de dinamometria de preensão palmar e preensão em pinça. Após 15 sessões de tratamento o grupo tratado com o ultrasom apresentou melhores resultados quando comparado com grupo tratado com o laser. A média da preensão palmar do grupo tratado com ultrasom foi de 36,6N, enquanto a média do grupo tratado com laser foi de $19,4 \mathrm{~N}$. Na preensão em pinça o grupo tratado com ultrasom teve média de $9,1 \mathrm{~N}$ enquanto o grupo tratado com laser teve média de 2,6N. No que diz respeito à diminuição do quando álgico e tendo em vista uma Escala Visual Analógica de $10 \mathrm{~cm}$, o grupo tratado com ultrasom teve redução média de 5,6cm na escala visual analógica, enquanto o grupo 
tratado com laser teve redução média de apenas $2,4 \mathrm{~cm}$. O ganho de força nestes pacientes é de suma importância para que estes readquiram funcionalidade com a mão acometida, possibilitando o retorno às suas atividades da vida diária (AVDs).

Yagci et al. (2009), objetivaram com seu estudo comparar o uso de órteses com a Laserterapia de baixa intensidade em conjunto à utilização órtese em pacientes diagnosticados com STC idiopática grave ou moderada. O desenho utilizado foi o randomizado controlado, e contou com 45 indivíduos. A amostra foi dividida em dois grupos: Grupo 1, composto por 24 pacientes, tratado apenas com a imobilização por órtese; e Grupo 2, composto por 21 pacientes, tratado com laserterapia de baixa intensidade mais a imobilização por órtese. Para um melhor "feedback" pós intervenção por parte dos pacientes, foram aplicados em ambos os grupos 2 questionários: o Questionário de Boston de sintomas severos e o Questionário de Boston de status funcional. O grupo 2 foi tratado com laser AsGaAl, cujo comprimento é $830 \mathrm{~nm}$, modulado com densidade de energia de $2 \mathrm{~J} / \mathrm{cm}^{2}$, densidade de potência de $50 \mathrm{~mW}$. A forma de aplicação foi pontual, sendo que o tempo de aplicação por ponto foi determinado pelo aparelho. Durante os três meses de acompanhamento, os indivíduos pertencentes ao grupo 2 passaram por 10 sessões de laserterapia associado à utilização da órtese, enquanto os indivíduos do grupo 1 tiveram apenas informações sobre o uso correto da órtese durante o mesmo período. No grupo tratado com órtese e laserapia 23,8\% dos pacientes relataram melhora total no quadro doloroso; $57,1 \%$ relataram melhora parcial, pois ainda sentiam dores intermitentes; e 19\% não perceberam nenhuma melhora. No grupo que utilizou apenas a órtese $4,2 \%$ relataram melhora total no quadro doloroso; $70,8 \%$ relataram melhora parcial, com permanência de dormência intermitente e dor; e $25 \%$ não perceberam nenhuma melhora.

Dincer et al. (2009), realizaram um estudo comparando o uso da órtese em conjunto à outras 2 técnicas de tratamento para a STC: a laserterapia de baixa intensidade e o ultra-som. Tal estudo contou com 50 pacientes do gênero feminino diagnosticas com STC bilateral. A amostra foi dividida em 3 grupo: grupo 1, controle, composto por 17 pacientes, submetidas apenas ao uso de órteses; grupo 2, composto por 15 pacientes, submetidas ao uso de órteses e ultra-som; grupo 3, composto por 18 pacientes, submetidas ao uso de órteses e laserterapia de baixa intensidade. O diagnóstico de STC foi confirmado a partir de ENMG. Foram utili- 
zados ainda 2 questionários para coleta de dados referente ao paciente: o Questionário de Boston de sintomas severos e o Questionário de Boston de status funcional. Durante o tratamento, todas as pacientes foram instruídas a utilizar a órtese durante a noite e em períodos em que os sintomas da STC de agravavam. O laser utilizado foi o AsGa-Diodo, cujo comprimento de onda é de $904 \mathrm{~nm}$, com densidade de energia de $1 \mathrm{~J} / \mathrm{cm}^{2}$, densidade de potência de $24 \mathrm{~mW}$. A forma de aplicação foi pontual perpendicular e em contato com a pele com tempo de tratamento de 30 segundos por ponto. Foram realizadas 10 sessões, sendo 1 por dia. Durante a aplicação os pacientes e o fisioterapeuta utilizaram óculos de proteção. Os grupos que utilizaram órtese em conjunto com o laser ou o ultrasom demonstraram melhores resultados quando comparados com o grupo que só utilizou a órtese como forma de tratamento. Dos 17 indivíduos que só utilizaram a órtese apenas 6 relataram diminuição no quadro álgico, enquanto os demais relataram não perceber diferença. Dos 15 que utilizaram o ultrasom junto à órtese, 10 relataram melhora da dor, mas 4 continuaram com parestesias intermitentes. Dos 18 que utilizaram o Laser junto à órtese 14 relataram melhora total do quadro álgico e parestésico. A aplicação do laser exige alguns cuidados, tanto para o paciente quanto para o terapeuta. Neste mesmo estudo, Dincer et al. (2009) enfatizaram que todos os pacientes e o fisioterapeuta utilizaram óculos de proteção durante as sessões de laserterapia. Trata-se de uma medida preventiva simples e eficiente a fim de se evitar os possíveis efeitos colaterais da irradiação do laser, mas que ainda é desrespeitada por muitos profissionais na prática clínica.

Pela descrição dos estudos de Dincer et al. (2009) e Yagci et al. (2009), notase a semelhança entre estes, onde os autores compararam o tratamento da STC baseado no uso de órteses com o tratamento de órtese em conjunto a outra técnica. Dincer et al. (2009) associaram a órtese à laserterapia em um grupo e ao ultrasom terapêutico em outro grupo e posteriormente comparou essa possibilidade com um grupo que utilizou apenas órtese como forma de tratamento. Já Yagci et al. (2009) formaram apenas dois grupos, sendo que um utilizou apenas órtese e o outro órtese associado à laserterapia. Em ambos os estudos os grupos que utilizaram apenas a órtese tiveram os piores resultados, sugerindo que os efeitos promovidos unicamente pela imobilização do túnel carpiano não são tão significativos como se esperava para a STC. Também em ambos os estudos os grupos que utilizaram o laser associado à imobilização por órteses obtiveram os melhores resultados no 
94 Universitas: Ciências da Saúde, Brasília, v. 7, n. 2, p. 85-96, 2009

João Paulo C. Cardozo, Léo Sebastião D. Filho, Hugo A. de Souza,

Flavia L. V. Dumas

que diz respeito à diminuição do quadro álgico e das parestesias associadas à compressão do nervo mediano.

Uma informação omitida na maioria dos estudos com utilização do laser e que influi diretamente nos resultados é a tonalidade da pele dos pacientes. Em função da maior quantidade de melanina, indivíduos de pele mais escura absorvem maior quantidade de radiação, logo, sugere-se que a aplicação do laser nesses pacientes seja mais efetiva que em pacientes de pele mais clara. Em se tratando da utilização do laser em patologias dermatológicas, este fato se torna ainda mais importante.

\title{
4 Conclusão
}

Mesmo a ação do laser sendo motivo de divergências entre alguns autores, foi possível observar com essa revisão que a laserterapia é eficiente no tratamento da STC, principalmente quando associada ao uso da órtese para o punho. Quando comparada com outras técnicas, não se mostrou superior.

\section{Applications of the low level laser therapy in the treatment of carpal tunnel syndrome}

\begin{abstract}
The objective of this study was to analyze, through literature review, the efficiency of low level laser therapy (LLLT) application in the treatment of the carpal tunnel syndrome. For this was made an articles review in the PUBMED and BIREME databases, published between 1985 and 2009, in English, Portuguese and Spanish. The most of papers showed that the LLLT is effective in the treatment of patients diagnosed with carpal tunnel syndrome. Concluded that, when associated with wrist's orthosis, the LLLT is an efficient method in the carpal tunnel syndrome treatment.
\end{abstract}

Keywords: Carpal Tunnel Syndrome. Laser therapy. Median nerve compression. 


\section{Referências}

ABLOVE, R. H.; ABLOVE, T. S. Prevalence of Carpal Tunnel Syndrome in Pregnant Women. Wisconsin Medical Journal, Wisconsin, v. 108, n. 4, p. 194-196, 2009.

BAKHTIARY, A. H.; RASHIDY-POUR, A. Ultrasound and laser therapy in the treatment of carpal tunnel syndrome. Australian Journal of Physiotherapy, Hawthorn, v. 50, p. 147-151, 2004.

BRAZ, A. G. et al. Tratamento da síndrome do túnel do carpo através da utilização da laserterapia. CONGRESSO BRASILEIRO DE ENGENHARIA BIOMÉDICA, 21., 2008, Salvador. Anais... Salvador: [s.n.], 2008. p.1155-1157.

DINCER, U. et al. The effectives of conservative treatments of Carpal Tunnel Syndrome: splinting, ultrasound and low-level laser therapies. Photomedicine and laser surgery, [S.1.], v. 27, n. 1, p. 119-125, 2009.

EKIM, A. et al. Effects of low level laser therapy in rheumatoid arthritis patients with carpal tunnel syndrome. Swiss Medical Weekly, [S.l.], v. 137, p. 347-352, 2007.

ELWAKIL, T. F. et al. Treatment of carpal tunnel syndrome by low-level laser versus open carpal tunnel release. Lasers Medical Scientific, [S.1.], v. 22, p. 265-270, 2007.

EVCIK, D. et al. Laser therapy in the treatment of carpal tunnel syndrome: a ramdomized controlled trial. Photomedicine and laser surgery, [S.1.], v. 25, n. 1, p.34-39, 2007.

GONZÁLEZ, J. L. et al. Tratamiento del syndrome del tunnel carpiano con laser He-Ne e infrarrojo: evaluación clinica y eletrofisiologica de los resultados. Revista Cubana de Ortopedia Traumatológica, Havana, v. 6, n. 2, p. 139-143, 1992.

KAROLCZAK, A. P. B.; VAZ, M. A.; MERLO, A. R. C. Síndrome do túnel do carpo. Revista Brasileira de Fisioterapia, Porto Alegre, v. 9, n. 2, p. 117-122, 2005.

KITCHEN, S. Eletroterapia: prática baseada em evidências. 11. ed. São Paulo: Manole, 2003.

KOUYOUMDJIAN, J. A. Síndrome do túnel do carpo: aspectos clínicoepidemiológicos em 668 casos. Arquivos de Neuropsiquiatria, [S.1.], v. 57, n. 2, p. 202-207, 1999.

STARKEY, C. Recursos terapêuticos em fisioterapia. São Paulo: Manole, 2001. 
96 Universitas: Ciências da Saúde, Brasília, v. 7, n. 2, p. 85-96, 2009 João Paulo C. Cardozo, Léo Sebastião D. Filho, Hugo A. de Souza, Flavia L. V. Dumas

VEÇOSO, M. C. Laser em fisioterapia. São Paulo: Lovise, 1993.

YAGCI, I. et al. Comparison of splinting and splinting plus low-level laser therapy in idiopathic carpal tunnel syndrome. Clinical Rehabilitation, [S.1.], v. 28, n. 9, p. 1059-1065, 2009. 